\title{
DIAGNÓSTICO E TRATAMENTO DE SIALOLITÍASE: REVISÃO DE LITERATURA
}

DIAGNOSIS AND TREATMENT OF SIALOLITHIASIS: LITERATURE REVIEW

\section{Natália Carneiro Fernandes}

Residente de Cirurgia Oral e Maxilofacial do Hospital Federal dos Servidores do Estado do Rio de Janeiro - HFSE e da Universidade Federal Fluminense - UFF.

\section{Fernanda Britto de Melo Silva}

Residente de Cirurgia Oral e Maxilofacial do Hospital Federal dos Servidores do Estado do Rio de Janeiro - HFSE e da Universidade Federal Fluminense - UFF.

\section{Rafael Seabra Louro}

Professor da disciplina de Cirurgia Bucal da Universidade Federal Fluminense - UFF, Niterói / RJ, Brasil.

\section{Marcelo José Pinheiro Guedes De Uzeda}

Professor da disciplina de Cirurgia Bucal da Universidade Federal Fluminense - UFF, Niterói / RJ, Brasil.

\section{Rodrigo Figueiredo de Brito Resende}

Professor da disciplina de Cirurgia Bucal da Universidade Federal Fluminense - UFF, Niterói / RJ, Brasil.

Instituição na qual o trabalho foi realizado: Faculdade de Odontologia da Universidade Federal Fluminense - Niterói/RJ

Categoria:Revisão de literatura

Informações do autor principal:

Natália Carneiro Fernandes

Estrada Eliseu de Alvarenga, 1471/508

Centro - Nilópolis - RJ, Brasil.

CEP: 26520-461

Email: natalia.1612@yahoo.com.br

Telefone: +5521993776246 


\title{
RESUMO
}

A sialolitíase corresponde a cerca da metade de todos os casos de doenças das glândulas salivares, tendo a submandibular comoo principal sítio acometido. Há uma maior prevalência em indivíduos do sexo masculino entre 30 e 60 anos de idade, com localização na porção distal do ducto e no hilo submandibulares e sendo de forma assintomática. Os sintomas se desenvolvem quando os cálculos atingem grandes proporções para ocasionar obstrução do fluxo salivar, onde é comum ocorreraumento de volume doloroso na região, especialmente durante as refeições. Em decorrência da obstrução, podem se desenvolver inflamação crônica ou infecção aguda e, mais raramente, abscessos. O objetivo deste trabalho, foi realizar uma revisão da literatura auxiliando ao profissional de Odontologia sobre o correto diagnóstico, tratamento e possíveis complicações da sialolitíase.

Palavras-chave: Cálculos das Glândulas Salivares; Glândula Submandibular.

\begin{abstract}
Sialolithiasis corresponds to about half of all cases of diseases of the salivary glands, with the submandibular as the main affected site. There is a higher prevalence in male individuals between 30 and 60 years of age, located in the distal portion of the submandibular duct and hilum and asymptomatic. Symptoms develop when the stones reach enough sizes to cause obstruction of the salivary flow, where it is common to report an increase in painful volume in the region, especially during meals. As a result of the obstruction, chronic inflammation or acute infection and, more rarely, abscesses may develop. The objective of this work was to carry out a literature review assisting the dental professional on the diagnosis, treatment and possible complications of sialolithiasis.
\end{abstract}

Key words: Salivary Gland Calculi; Submandibular Gland.

\section{INTRODUÇÃO}

A sialolitíase corresponde a cerca de metade de todos os casos de doenças das glândulas salivares. A glândula submandibular é o principal sítio acometido, concentrando de 80 a 90\% de todos os casos(ZHAO YN, ZHANG YQ. et al. 2019; COMBES J, KARAVIDAS K. et al. 2009; WILLIAMS MF. 1999). Os sialolitos estão mais frequentemente localizados na porção distal do ducto e no hilo submandibulares, e 
a idade média no momento do diagnóstico é de 30 a 60 anos. Há uma incidência maior entres os indivíduos do sexo masculino (ZHAO YN, ZHANG YQ. et al. 2019; WILLIAMS MF. 1999; DELLI K, SPIJKERVET FK. et al. 2014; HAMMETT JT, WALKER C. 2019).

Os sialolitos submandibulares são usualmente maiores que os encontrados nas glândulas parótidas ou salivares menores e costumam ser unitários. Em apenas aproximadamente $5 \%$ dos casos existem três ou mais sialolitos. O envolvimento bilateral das glândulas submandibulares ou o acometimento simultâneo de múltiplas glândulas são raros, bem como o desenvolvimento de sialolitos em pacientes pediátricos (WILLIAMS MF. 1999).

A maior frequência de sialolitos nas glândulas submandibulares em comparação às demais glândulas salivares é devida a algumas características únicas desta glândula. Seu ducto largo, longo e de trajeto ascendente, em conjunto com o fluxo lento de saliva, favorecem a estase salivar e a deposição de sais. A estase salivar também é favorecida pela maior viscosidade da saliva produzida por esta glândula, que é rica em mucina. Além disso, seu pH é mais alcalino que da saliva produzida pelas outras glândulas, o que predispões a precipitação de componentes inorgânicos e a formação de cálculos (WILLIAMS MF. 1999; HAMMETT JT, WALKER C. 2019).

A presença de sialolitos pode ser assintomática. Os sintomas se desenvolvem quando os cálculos atingem tamanhos suficientes para ocasionar obstrução do fluxo salivar. Nesta situação, é comum o relato de aumento de volume doloroso na região submandibular, especialmente após as refeições. Em decorrência da obstrução, podem se desenvolver inflamação crônica ou infecção aguda e, mais raramente, abscessos.A sialolitíase pode se manifestar também como um aumento de volume indolor ou dor locar sem aumento de volume associado (COMBES J, KARAVIDAS K. et al. 2009; WILLIAMS MF. 1999; HAMMETT JT, WALKER C. 2019).

O curso natural da sialolitíase envolve o desenvolvimento de sialadenite (aguda ou crônica) e atrofia do tecido glandular. Se não houver tratamento, o dano tecidual evolui comfibrose e perda completa da função secretora e, consequentemente, do fluxo salivar não estimulado (WILLIAMS MF. 1999; HAMMETT JT, WALKER C. 2019). 


\section{REVISÃO DA LITERATURA}

\section{Fisiopatologia}

A real causa e o mecanismo de desenvolvimento dos sialolitos ainda são desconhecidos, embora muitas teorias tenham sido elaboradas. Acredita-se que carcaterísticas anatômicas e funcionais da glândula, bem como a composição salivar tenham participação na sialolitíase (WILLIAMS MF. 1999; HAMMETT JT, WALKER C. 2019; Kao WK, Chole RA. et al. 2020).

Sua etiologia pode estar relacionada a sialadenite crônica. Em uma glândula inflamada surgem pequenos cálculos denominados microlitos. Estes causammicroobstruções, que favorecem o surgimento de sialadenite crônica, alterações no fluxo, na composição salivar e estase. O resultado é a formação de um gel, que atua como uma matriz para a deposição de sais e componentes orgânicos, formando cálculos. Outro postulado propõe que a participação dos microlitos se dá de maneira diferente, através da atuação direta destes como estrutura base para a formação de sialolitos(WILLIAMS MF. 1999; HAMMETT JT, WALKER C. 2019; KAO WK, CHOLE RA. et al. 2020).

As micro-osbtruções por microlitostamném podem ser subsequentes à diminuição da atividade secretora. A diminuição da secreção salivar permite o acúmulo de microlitos, que em condições normais seriam expelidos com o fluxo salivar (KAO WK, CHOLE RA. et al. 2020).

Alterações na composição salivar também são supostamente responsáveis pela formação de sialolitos. Uma das hipóteses para a etiologia da sialolitíase propõe que um fenômeno metabólico desconhecido acarreta um aumento da concentração de bicarbonato na saliva, reduzindo a solubilidade do fosfato de cálcio e induzindo a precipitação de íon cálcio e fosfato (WILLIAMS MF. 1999;KAO WK, CHOLE RA. et al. 2020).

Uma outra hipótese é de que a sialolitíase tenha um caráter multifatorial. Esta propõe que bactérias da cavidade oral alcançam a glândula e formam um biofilme, estimulando uma resposta inflamatória. $\mathrm{O}$ complexo formado pelos microrganismos e 
células de defesa do hospedeiro atua como uma matriz para a precipitação de cálcio e fomação de sialolitos (HAMMETT JT, WALKER C. 2019).

Alguns estudos tentam correlacionar a sialolitíase a condições sistêmicas e ao uso de medicamentos. Não foi encontrada relação entre a formação de cálculos salivares e alterações no metabolismo do cálcio. Entretanto, parece haver relação entre a existência de gota e uma predisposição ao desenvolvimento de sialolitos. Nestes pacientes, entretanto, os cálculos têm uma composição diferente, rica em ácido úrico. Também foi sugerida uma possível associação entre a sialolitíase e a nefrolitíase. Quanto ao uso de medicamentos, algumas classes farmacológicas sabidamente causam redução na produção de saliva e, portanto, podem teoricamente estar associada ao aumento de formação de sialolitos. Dentre estas classes estão alguns anti-histamínicos, anti-hipertensivos, antidepressivos e antipsicóticos. No entanto, não há uma relação clara e direta estabelecida entre o uso destas drogas e a sialolitíase (WILLIAMS MF. 1999).

\section{Diagnóstico}

O diagnóstico de sialolotíase pode ser muitas vezes estabelecido com uma anamnese e um exame físico cuidadosos. A palpação bimanual do soalho oral e da glândula submandibular é uma manobra útil para identificar cálculos. Além disso, uma glândula com textura firme e pétrea sugere perda de função e fibrose (HAMMETT JT, WALKER C. 2019). Para o diagnóstico diferencial de neoplasias, exames de imagem para a identificação de cálculos não palpáveis e a punção aspirativa por agulha fina (PAAF) podem ser úteis (WILLIAMS MF. 1999; HAMMETT JT, WALKER C. 2019).

A inspeção pode mostrar assimetria facial, com aumento de volume da glândula acometida. Alguns cálculos localizados no ducto submandibular podem ser visualizados na cavidade oral (HAMMETT JT, WALKER C. 2019).Casos envolvedosialolitos localizados na porção proximal do ducto ou intragladulares normalmente necessitam de exames de imagem complementares para diagnóstico(HAMMETT JT, WALKER C. 2019). 


\subsection{Radiografias}

A maioria dos cálculos salivares da glândula submandibular são visíveis em exames radiográficos. Os mais úteis nas investigações de sialolitíase submandibular são radiografias oclusais e panorâmicas (WILLIAMS MF. 1999).

Entretanto, outras alterações podem produzir imagens radiopacas na mesma região que os sialolitos, comoflebólitos nas veias linguais, linfonodos cervicais calcificados e aterosclerose da artéria lingual. Exames de tomografia computadorizada ou sialografia são úteis para o diagnóstico diferencial (WILLIAMS MF. 1999).

Embora sejam técnicas simples e de fácil acesso, as radiografias são pouco sensíveis, e pequenos cálculos ou aqueles pouco calcificados podem passar despercebidos. Portanto, estes exames podem ser utilizados em uma investigação inicial, mas frequentemente não são suficientes para o diagnóstico (WILLIAMS MF. 1999; DELLI K, SPIJKERVET FK. et al. 2014; HAMMETT JT, WALKER C. 2019).

\subsection{Sialografia}

Uma das técnicas mais úteis na avaliação de glândulas salivares é a sialografia. O exame é realizado pela injecção de contraste através do óstio do ducto da glândula, promovendo o preenchimento retrógrado do sistema ductal. Em seguida, são obtidas imagens radiográficas. Quando utilizadas radiografias digitais, é possível visualizar até mesmo ramos quaternários e o parêquima glandular (WILLIAMSMF. 1999; DELLI K, SPIJKERVET FK. et al. 2014; HAMMETT JT, WALKER C. 2019)

A sialografia permite a identificação e localização de cálculos e constriç̧õesductais. Além disso, o contraste injetado atua como sialogogo e, portanto, pode auxiliar na eliminação de pequenos sialolitos. O sialograma também fornece informações sobre a função glandular, através da observação de dilatação dos ductos. Este exame também é útil na pré-avaliação de pacientes para a endoscopia (WILLIAMS MF. 1999; HAMMETT JT, WALKER C. 2019).

No entanto, esta técnica possui limitações e desvantagens consideráveis. Ela está contraindicada em indivíduos com histórico de alergia a contraste e em casos em que o 
sialolito está localizado na porção distal do ducto, devido ao risco de deslocamento do cálculo para uma posição menos favorável à remoção. Também não deve ser empregada em casos de infecção aguda. Outro ponto negativo é a exposição à radiação para a aquisição de imagens. A sensibilidade e a especificidade são variáveis, atingindo de $64 \%$ a $100 \%$ e de $88 \%$ a $100 \%$, respecivamente (WILLIAMS MF. 1999; DELLI K, SPIJKERVET FK. et al. 2014; HAMMETT JT, WALKER C. 2019).

\subsection{Ultrassonografia}

A ultrassonografia (USG) consiste em um método não-invasivo para o estudo das glândulas salivares, que assim como a sialografia permite a identificação e localização de sialolitos. Nas imagens obtidas, estes aparecem como focos hiperecogênicos com sombra acústica posterior associada (WILLIAMS MF. 1999; HAMMETT JT, WALKER C. 2019).

Além de não ser invasiva, a USG é uma técnica relativamente barata, amplamente disponível e isenta de exposição à radiação. É especialmente útil para pacientes alérgicos a contraste e em casos de infecção aguda, nos quais a sialografia está contraindicada (HAMMETT JT, WALKER C. 2019).

Entretanto, seus resultados são altamente dependentes do operador, o que torna a sensibilidade e a especificidade bastante variáveis. Em geral, elas são mais altas para sialolitos acima de 2 a $3 \mathrm{~mm}$, atingindo níveis comparáveis aos da tomografia computadorizada sem contraste e da endoscopia (WILLIAMS MF. 1999; DELLI K, SPIJKERVET FK. et al. 2014; HAMMETT JT, WALKER C. 2019).

\subsection{Tomografia Computadorizada (TC)}

Nos casos em que existe a suspeita de sialolitos muito pequenos, a TC emerge como uma técnica extremamente útil. A maioria dos cálculos são mineralizados o suficiente para serem visíveis através de TC. Quando associada à administração intravenosa de contraste, a TC permite uma visualização rica em detalhes tanto do 
sistema ductal quanto do parênquima glandular (WILLIAMS MF. 1999; DELLI K, SPIJKERVET FK. et al. 2014; HAMMETT JT, WALKER C. 2019).

Dentre as suas vantagens estão sensibilidade e especificidade excelentes e uma ampla disponibilidade. Entretanto, apresenta um custo elevado e significativa exposição à radiação (HAMMETT JT, WALKER C. 2019). Atualmente, a TC do tipo cone beam representa uma boa opção à TC convencional, uma vez que fornece resultados semelhantes com menor custo e uma exposição à radiação cerca de dez vezes menor (DELLI K, SPIJKERVET FK. et al. 2014).

\subsection{RessonânciaNuclear Magnética (RNM)}

A RNM é uma técnica alternativa para a investigação de sialolitíase, com as vantagens de não serinvasiva e ser altamente eficiente. Embora não permita a visualização dos cálculos, estes podem ser identificados indiretamente pela dilação do ducto salivar onde se encontram. Este método pode ser utilizado em pacientes com quadros infecciosos agudos em curso, e permite a diferenciação dos quadros agudos e crônicos com base na intensidade de sinal (WILLIAMS MF. 1999; DELLI K, SPIJKERVET FK. et al. 2014).

A sensibilidade e a especificidade da ressonância magnética são comparáveis às da TC, com a vantagem de fornecer imagens precisas dos ductos e parênquima sem injeção de contraste e sem exposição à radiação. Entretanto, trata-se de um exame de alto custo e com disponibilidade inferior à TC e à USG (HAMMETT JT, WALKER C. 2019).

\subsection{Sialoendoscopia}

Quando as técnicas mencionadas anteriormente não são suficientes para a identificação dos sialolitos, a sialoendoscopia com finalidade diagnóstica é uma ferramenta de grande utilidade. Esta técnica permite a visualização direta de sialolitos, independente de seu grau de mineralização, de plugs mucosos e do sistema ductal. Além disso, através da abordagem endoscópica é possível realizar diagnóstico e tratamento simultaneamente (DELLI K, SPIJKERVET FK. et al. 2014; HAMMETT JT, WALKER C. 2019). 


\section{Formas de tratamento}

Múltiplas modalidades terapêuticas estão disponíveis para o manejo da sialolitíase. As possibilidades variam de terapias conservadoras à exérese da glândula, e a escolha do tratamento a ser empregado é dependente principalmente da localização, do tamanho e do número de sialolitos existentes. Também é importante considerar tratamentos prévios e a condição sistêmica do paciente, bem como sua capacidade de tolerância a tratamentos sob anestesia local (ZHAO YN, ZHANG YQ. et al. 2019; DELLI K, SPIJKERVET FK. et al. 2014; HAMMETT JT, WALKER C. 2019).

\subsection{Tratamento Conservador}

O tratamento inicial da sialolitíase deve consistir de terapias conservadoras, especialmente nos casos envolvendo pequenos sialolitos. O objetivo destas é aumentar o fluxo salivar, na tentativa de que o cálculo seja expelido (WILLIAMS MF. 1999).

$\mathrm{O}$ tratamento conservador inclui o aumento na ingestão hídrica e de alimentos ácidos, aplicação de calor local, massagem da glândula e administração de sialogogos e anti-inflamatórios não-esteroidais. Caso existam sinais de infecção, deve ser realizada também terapia antibiótica (WILLIAMS MF. 1999; DELLI K, SPIJKERVET FK. et al. 2014; HAMMETT JT, WALKER C. 2019).

Se a abordagem conservadora não for eficaz na resolução da condição, outra modalidade terapêutica deve ser adotada (HAMMETT JT, WALKER C. 2019).

\subsection{SialolitectomiaTransoral}

Sialolitos que estejam localizados na porção anterior do ducto submandibular, especialmentes aqueles que estão a até $2 \mathrm{~cm}$ do óstio, são passíveis de ressecção por abordagem transoral (WILLIAMS MF. 1999).

O ducto submandibular e seuóstio podem ser distendidos consideravelmente sem que haja danos a estas estruturas, permitindo a saída de pequenos cálculos (WILLIAMS 
MF. 1999). Sendo assim, pacientes que apresentam sialolitos com tamanho inferior a 2 $\mathrm{mm}$ e localizados na porção mais anterior do ducto submandibular (até $1 \mathrm{~cm}$ do óstio) podem ser beneficiados pela remoção destes através do ducto (WILLIAMS MF. 1999; SCHMITZ S, ZENGEL P.et al. 2008).

Este procedimento é realizado sob anestesia local, através da inserção de cânulas lacrimais de calibres progressivamente maiores, dilatando o ducto e possibilitando que o cálculo seja removido por ordenha (WILLIAMS MF. 1999).

Uma modificação desta técnica consiste em, após identificação do sialolito com a ponta da cânula ou através de palpação, acessar o ducto e incisá-lo para a visualização direta e remoção do cálculo. Esta técnica é útil para cálculos localizados um pouco menos anteriormente, entre 1 a $2 \mathrm{~cm}$ de distância do óstio. Nesta abordagem é necessário cuidado com o nervo lingual, que está intimamente relacionado ao ducto em sua porção posterior. Não é necessário realizar síntese da ferida, e nenhum tratamento é necessário caso surja uma fístula salivar no local (WILLIAMS MF. 1999).

Sialolitos submandibulares em porções mais profundas do ducto submandibular podem ser passíveis de remoção atravésde abordagem transoral sob anestesia geral. $\mathrm{O}$ ducto submandibular é dissecado, enquanto o nervo lingual deve ser identificado e protegido. O ducto é então aberto na localização do cálculo, que é diretamente visualizado e removido. É realizada então a síntese do ducto e do soalho (COMBES J, KARAVIDAS K. et al. 2009; WILLIAMS MF. 1999).

A abordagem transoral permite que a glândula submandibular seja preservada, $o$ que representa uma vantagem em comparação à sialadenectomia. No entanto, não se trata de uma técnica isenta de riscos. Os vasos sublinguais e o nervo lingual então próximos ao ducto submandibular e, portanto, podem ocorrer hemorragia e parestesia lingual (ZHAO YN, ZHANG YQ. et al. 2019; COMBES J, KARAVIDAS K. et al. 2009).

Pacientes tratados por abordagem transoral apresentam taxa de recidiva de aproximadamente $18 \%$. Estas podem ser verdadeiras ou tratar-se de cálculos residuais, que não foram devidamente ordenhados no transoperatório(WILLIAMS MF. 1999). 


\subsection{Sialoendoscopia}

Atualmente, a sialoendoscopia consiste em uma alternativa para a exérese de sialolitos localizados profundamente, na porção proximal do ducto submandibular ou intraglandular. Esta técnica representa uma opção à sialadenectomia em alguns casos, permitindo que a ressecção total da glândula fique restrita a casos onde não foi obtido sucesso com a abordagem endoscópica ou em que há severo comprometimento da função glandular (ZHAO YN, ZHANG YQ. et al. 2019; HAMMETT JT, WALKER C. 2019).

Os cálculos salivares podem ser removidos íntegros ou fragmentados previamente através do ducto submandibular. A fragmentação pode ser obtida por ação mecânica através de fórceps ou microdrill. Também é possível realizar litotripsiaintracorpóreasultrassônica, a laser ou pneumática (Koch M, Schapher M. et al. 2018).

As principais vantagens da sialoendoscopia são a menor possibilidade de complicações e a possibilidade de realização sob anestesia local (HAMMETT JT, WALKER C. 2019).

\subsection{Litotripsia Extracorpórea por Onda de Choque (LECO)}

A litotripsia extracorpórea por onda de choque (LECO) é uma alternativa para sialolitos não acessíveis por via endoscópica.Na LECO, ondas de choque induzem a fragmentação do cálculo, e os fragmentos podem ser carreados até o óstio pelo fluxo salivar. Esta metodologia é uma adaptação da utilizada para o tratamento dos cálculos renais (HAMMETT JT, WALKER C. 2019).

A LECO tem um caráter minimamente invasivo, sem importantes complicações relatadas. No entanto, apresenta eficácia limitada, comtaxa de sucesso inferiores a 50\% na maioria dos estudos.Seu sucesso também é dependente das dimensões do sialolito, sendo normalmente ineficaz para aqueles com tamanho a partir de $8 \mathrm{~mm}$ (ZHAO YN, 
ZHANG YQ. et al. 2019; COMBES J, KARAVIDAS K. et al. 2009; SCHMITZ S, ZENGEL P. et al. 2008; KOCH M, SCHAPHER M. et al. 2018).

A associação da LECO à sialoendoscopia permite aumentar a capacidade de resolução da siaolitíase submandibular. Os cálculos podem ser fragmentados e deslocados anteriormente até uma localização que permita sua visualização através de endoscopia. Os fragmentos podem ser, então, removidos por via endoscópica (KOCH M, SCHAPHER M. et al. 2018).

\subsection{Sialadenectomia}

Classicamente, casos de sialolitíase submandibular sintomáticos não responsivos ao tratamento conservador e cujos sialolitos estão localizados no interior da glândula ou no ducto proximal são tratados pela exérese da glândula submandibular afetada (ZHAO YN, ZHANG YQ. et al. 2019; COMBES J, KARAVIDAS K. et al. 2009; SCHMITZ S, ZENGEL P.et al. 2008).

Alguns autores defendem que o ducto submandibular deve ser ressecado em conjunto com a glândula submandibular associada. Esta ideia é sustentada pela possibilidade de desenvolvimento de novos sialolitos no ducto remanescente, além da ocorrência de inflamação desta estrutura no pós-operatório em cerca de $5 \%$ dos pacientes. Outra justificativa para a remoção do ducto é o possível desenvolvimento de cisto de retenção na glândula sublingual ipsilateral, em decorrência da obstrução dos ductos de Rivinusque drenam para o submandibular (WILLIAMS MF. 1999).

A redução do fluxo salivar não estimulado é uma consequência da sialadenectomia. Cerca de $57 \%$ dos pacientes relatam xerostomia após serem submetidos ao procedimento. Considerando a possibilidade de recuperação funcional da glândula com a remoção do sialolito em $75 \%$ dos casos, atualmente a sialadenectomia deve ser mantida como última possibilidade terapêutica. Além disso, esta técnica está associada a risco de lesão transitória e permanente dos nervos lingual e marginal da mandíbula, e resulta em uma cicatriz potencialmente inestética (ZHAO YN, ZHANG YQ. et al. 2019; WILLIAMS MF. 1999) 


\section{CONSIDERAÇÕES FINAIS}

A sialolitíase representa uma importante causa de desordens salivares. Esta condição apresenta um bom prognóstico, e a maioria dos pacientes responde bem a tratamentos conservadores. Atualmente múltiplos procedimentos minimamente invasivos foram desenvolvidos, apresentando taxas de sucesso satisfatórias com pouca morbidade associada.

Entretanto, estas modalidades terapêuticas demandam disponibilidade de recursos técnicos e pessoal capacitado. Além disso, o longo tempo entre o surgimento dos sintomas, o diagnóstico e o tratamento faz com que muitos pacientes apresentem quadros muito evoluídos, com fibrose glandular severa e sialolitos de grandes dimensões, limitando a aplicabilidade das técnicas menos invasivas. Desta forma, a sialadenectomia permanece como um tratamento amplamente utilizado na saúde pública, apesar de apresentar riscos e morbidade significativos.

\section{REFERÊNCIAS BIBLIOGRÁFICAS}

1. Zhao YN, Zhang YQ. et al. Treatment strategy of hilar and intraglandular stones in wharton's duct: A 12-year experience. Laryngoscope. 2019 Nov 6. doi: 10.1002/lary.28361 [Epub ahead of print].

2. Combes J, Karavidas K. et al. Intraoral removal of proximal submandibular stones - an alternative to sialadenectomy? Int J Oral MaxillofacSurg, 38(8): 813-816, ago

2009.

3. Williams MF. Sialolithiasis. OtolaryngolClin North Am, 32(5): 819-34, out 1999.

4. Delli K, Spijkervet FK. et al. Salivary gland diseases: infections, sialolithiasis and mucoceles. Monogr Oral Sci. 24: 135-48, 2014. 
5. Hammett JT, Walker C. Sialolithiasis. SourceStatPearls [Internet]. Treasure Island (FL): StatPearls Publishing, out 2019.

6. Kao WK, Chole RA. et al. Evidence of a microbial etiology for sialoliths. $\begin{array}{llll}\text { Laryngoscope. } & \text { 130(1): } & 69-74, & \text { jan }\end{array}$

7. Schmitz S, Zengel P. et al. Long-term evaluation of extracorporeal shock wave lithotripsy in the treatment of salivary stones. J LaryngolOtol. 122(1): 65-71, jan 2008.

8. Koch M, Schapher M. et al. Multimodal treatment in difficult sialolithiasis: Role of extracorporeal shock-wave lithotripsy and intraductal pneumatic lithotripsy. Laryngoscope. 128(10): E332-E338, out 2018. 